amino acid metabolism

glycine

hyperglycinemia isovaleric acidemia ketoacidosis

\title{
Isovaleric Acidemia Presenting with Altered Metabolism of Glycine
}

\author{
Toshiyuki Ando, William G. Klingberg, Arthur N. Ward, Karsten Rasmussen, \\ and William L. Nyhan ${ }^{[36]}$ \\ Department of Pediatrics, University of California, San Diego, La Jolla, California, and Department of Pediatrics, \\ West Virginia University, Morgantown, West Virginia, USA
}

\begin{abstract}
Extract
A 7-year-old girl with periodic acidosis, lethargy, and coma beginning during the 1st year of life was studied. Episodes of ketoacidosis usually occurred with minor respiratory or gastrointestinal infections. There was history neither of peculiar body odor nor characteristic smell like that of sweaty feet. Hyperglycinemia, $4.2 \mathrm{mg} / 100 \mathrm{ml}$, and hyperglycinuria, $361 \mathrm{mg} / \mathrm{liter}$, were found at times of illness with acidosis. A clinical diagnosis of ketotic hyperglycinemia was made in 1966. Glycine metabolism was therefore investigated in vivo using ${ }^{14} \mathrm{C}$-labeled glycine. The oxidation of glycine to respiratory $\mathrm{CO}_{2}$ and formation of serine from glycine were found to be normal. The labeling of urinary hippurate, however, the largest component of the labeled products of glycine in the urine in normal individuals, was virtually zero. An abnormal, labeled product of glycine was found in the urine and identified as isovalerylglycine. As much as $6 \%$ of the ${ }^{14} \mathrm{C}$-glycine administered was incorporated into urinary isovalerylglycine within $24 \mathrm{hr}$. Isovalerylglycine in urine was $2880 \mathrm{mg} / 24 \mathrm{hr}$, while normal individuals excrete less than $2 \mathrm{mg} / 24 \mathrm{hr}$. Isovaleric acid was found in both urine and plasma. Isovaleric acid in the plasma and urine, $2.86 \mathrm{mg} / \mathrm{liter}$ and $2.56 \mathrm{mg} / \mathrm{day}$, respectively, was only five to six times larger than that found in normal individuals. Formation and excretion of isovalerylglycine may be a compensatory mechanism for the elimination of toxic amounts of isovaleric acid from the body. Biosynthesis of such huge amounts of isovalerylglycine may alter the overall metabolism of glycine. Isovaleric acidemia may present with hyperglycinemia and hyperglycinuria. Examination of the urine for isovalerylglycine is a convenient method for detecting the syndrome of isovaleric acidemia.
\end{abstract}

\section{Speculation}

Isovaleric acidemia, in common with propionic acidemia with ketotic hyperglycinemia and methylmalonic acidemia, produces dramatic episodes of ketoacidosis. It is now clear that, like these conditions, isovaleric acidemia can also produce leukopenia and elevated concentrations of glycine in body fluids. The mechanisms causing these abnormalities are unknown. Elucidation of metabolic interfaces among these conditions could lead to understanding of the pathophysiology involved.

\section{Introduction}

An increasing number of disorders have been recognized in which serious or overwhelming illness may occur very early in life. The first of these to be recognized was what was originally called idiopathic hyperglycinemia [7] and later ketotic hyperglycinemia to dis- 
tinguish it from the nonketotic form [2]. Methylmalonic acidemia can also produce the biochemical phenotype and clinical manifestations of ketotic hyperglycinemia [13]. Clinical manifestations of overwhelming neonatal illness have been described in isovaleric acidemia [18] and propionic acidemia [10]. The clinical presentations of these conditions resemble one another closely. It is now recognized that propionic acidemia is a prominent feature of what has been called ketotic hyperglycinemia $[3,8]$. It is not yet clear how much heterogeneity exists among these conditions.

Very few patients with isovaleric acidemia have been reported, but the characteristics of the illness have been well documented [5, 18-20]. Hyperglycinemia and hyperglycinuria have not previously been observed in this condition.

This report describes a girl with isovaleric acidemia in whom the course of illness after infancy was relatively mild. There was no history of a peculiar body odor. She presented with modest hyperglycinemia and leukopenia and was found to have isovaleric acidemia and to produce isovalerylglycine as a product of glycine metabolism.

\section{Case Report}

Patient TM was a 7-year-old schoolgirl with periodic acidosis, modest hyperglycinemia, and neutropenia. She was admitteed to the Clinical Center for Metabolic Studies of the West Virginia University Hospital in December, 1967.

She had been born at term weighing $6 \mathrm{lbs}, 12 \mathrm{oz}$. The neonatal period was uneventful, and breast feeding was initiated. However, she ate very poorly and lost weight; by $1-2$ months she was said to weigh $2-3$ 1bs. She was hospitalized in Ohio for 5-6 months and fed bananas and cottage cheese, and she regained her birth weight. During the lst year of life she had her first attack of dehydration, lethargy, and coma. Recovery followed vigorous treatment with parenteral fluids. Attacks were repeated two to three times a year during the first few years of her life. The duration of coma varied from a few hours to 7 days. A number of attacks followed acute infectious diseases such as pharyngitis, otitis media, or gastroenteritis.

She was first admitted to the West Virginia University Hospital at 2 years of age with pneumococcal pneumonia and sepsis. Subsequently she has had three hospital admissions, each with a sinilar picture. Fever was followed by vomiting, dehydration, and acidosis. She exhibited Kussmaul breathing and was usually quite dehydrated during attacks. During the ketotic attacks the serum $\mathrm{CO}_{2}$ content was generally 10-12 $\mathrm{mEq} /$ liter, and acetone was regularly found in the urine. Long-chain ketones were not found in the urine. During some of her ketotic episodes she was noted to have leukopenia (white blood cell count, 2700-5000). On each occasion she responded to parenteral fluid therapy.

Parents and three siblings were living and well; an older brother had died at 13 months in an acute attack of illness which the mother felt was very similar to that of the patient.

In the case of $T M$ a possible relation of acute attacks to salicylate administration was suggested as aspirin had been given 12-48 $\mathrm{hr}$ prior to each episode of dehydration and acidosis after febrile status appeared.

At the time of the study she had been well for over 6 months. Her height, 44.7 inches, was below the 3rd percentile, and she weighed 37.4 lbs. She was slightly microcephalic. Physical examination was otherwise negative. At no time did she or her brother have an odor which resembled sweaty feet, although with acute episodes each clid, in retrospect, "smell sour" to the mother.

The electroencephalogram showed some slowing and spikes in sleep. The concentration of glycine in the serum at a time of good health 10 months before admission was $3.2 \mathrm{mg} / 100 \mathrm{ml}$. During an episode of ketosis 3 months later it was $4.2 \mathrm{mg} / 100 \mathrm{ml}$. At this time the concentration of glycine in the urine was $361 \mathrm{mg} /$ liter. By contrast, the concentrations of alanine and threonine in the urine were 76 and $33 \mathrm{mg} /$ liter, respectively. There was no methylmalonic acid in the urine.

\section{Methods}

Concentrations of amino acids in plasma and urine were determined using an automatic amino acid analyzer [24]. The metabolism of glycine was studied in vivo during a time of remission. The design of the experiment permitted an assessment of the metabolism of each of the carbons of glycine, following the separate intravenous injection of glycine- $1-{ }^{14} \mathrm{C}$ and glycine${ }^{2.14} \mathrm{C}\left(2.0 \mu \mathrm{Ci} / \mathrm{kg}\right.$; glycine-1. ${ }^{14} \mathrm{C}, 6.34 \mathrm{mCi} / \mathrm{mmole}[25]$; and glycine-2-14 $\mathrm{C}, 21.5 \mathrm{mCi} / \mathrm{mmole}[26])$. The materials, procedures, and analytical methods employed in these studies have previously been described [2].

Urinary hippuric acid was analyzed by the method of Chantrenne [6]. Radioactive products of glycine excreted in the urine were monitored in the effluent from the long column of the amino acid analyzer using a continuous flow monitor [27].

Identification of the unknown product of glycine as 


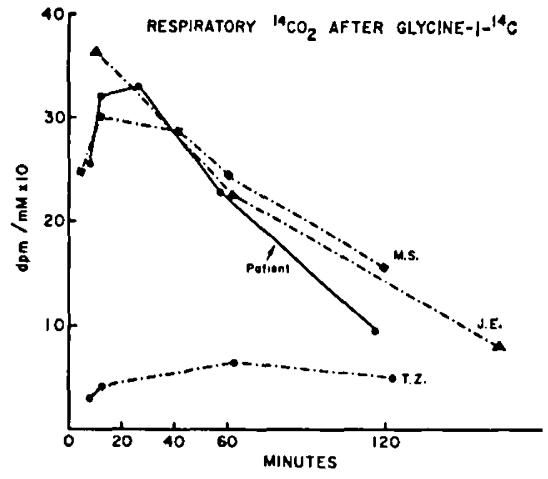

Fig. 1. Formation of ${ }^{14} \mathrm{CO}_{2}$ from glycine-1 ${ }^{14} \mathrm{C}$ in the patient and three other individuals. $M S$ and $J E$ were control individuals previously reported (2), and $T Z$ had nonketotic hyperglycinemia (2).

isovalerylglycine was confirmed by the methods described by Tanaka and Isselbacher [19] using silicic acid column chromatography, gas-liquid chromatography, infrared spectrometry, and mass spectrometry of the purified isovalerylglycine. $\mathrm{N}$-propionyl-, N-butyryl-, $\mathrm{N}$-valeryl-, and $\mathrm{N}$-isovalerylglycine were synthesized by the method of Bondi and Eissler [4]. These acylglycines were used as reference standards for identification and quantitation of isovalerylglycine in the patient's urine. A gas chromatograph [28] equipped with a hydrogen flame detector was used for all gas chromatographic analyses. The short-chain organic acids of the plasma were analyzed using the procedures of Tanaka $e t a l$. [18]. Urinary isovaleric acid was determined using a simple gas chromatographic procedure with a Porapak [29] column [14].

\section{Results}

\section{Metabolism of Glycine-1-14 $C$ and Glycine-2.14 $C$}

The metabolism of each of the carbon atoms of glycine was assessed following the separate intravenous injection of glycine- $1 .{ }^{14} \mathrm{C}$ and of glycine-2-14 $\mathrm{C}$. At the time of the experiment the patient had been well for an extended period of time during which the glycine concentration of her plasma varied from 2.34 to 2.91 $\mathrm{mg} / 100 \mathrm{ml}$. An oral serine loading test demonstrated ready conversion of serine to glycine. The conversion of glycine- $1-{ }^{14} \mathrm{C}$ to respiratory $\mathrm{CO}_{2}$ is illustrated in Figure 1 . The curve obtained in the patient was virtually identical to those of the two control individuals ( $M S$ and $J E$ ) and very different from that of the patient with nonketotic hyperglycinemia, $T Z$ [2]. The oxidation of glycine-2-14 $\mathrm{C}$ by patient $T M$ was also normal.

The conversion of glycine-2-14 $\mathrm{C}$ to serine is shown in Table I. The specific activities of the glycine isolated from the plasma at the various time intervals were quite similar to those previously determined in control individuals [2], indicating that the pool sizes of glycine were comparable. There was rapid conversion of glycine to serine as indicated by the isolation of labeled serine within 5 min of the injection of labeled glycine. The specific activities of the labeled serine were quite similar to those of controls [2]. Furthermore, when the serine was degraded and carbon 3 was analyzed specifically, the isotope content was virtually identical with those of control individuals. Thus, decarboxylation of glycine and the conversion of the second carbon of glycine to the third carbon of serine were found to be normal in the patient.

\section{Diminished Labeling of Hippuric Acid}

During the analyses of the labeled amino acids from plasma and urine, a radioactive peak which usually appears directly after serine in analysis of plasma from control individuals was missing (Fig. 2). A small amount of labeled glycine was found in the urine of both patient and control subject. The major radioactive product of glycine in controls was in a peak that followed serine and chromatographed with glutamine. In the patient samples virtually no isotope was found in this area. The radioactive peak found in the urine of control individuals was identified as hippuric acid.

The labeled hippurate peak chromatographed separately from glutamine in some runs and did not react

Table I. Isotope content of glycine and serine and carbon 3 of serine in plasma ${ }^{1}$

\begin{tabular}{|c|c|c|c|c|c|c|c|}
\hline \multirow{2}{*}{ Time, $\min$} & \multirow{2}{*}{ 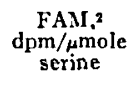 } & \multicolumn{3}{|c|}{ Serine } & \multicolumn{3}{|c|}{ Glycine } \\
\hline & & $\mu$ moles/liter & $\mathrm{dpm} / \mu \mathrm{mole}$ & $\mathrm{dpm} / \mathrm{ml}$ & $\mu$ moles/liter & $\mathrm{dpm} / \mu \mathrm{mole}$ & $\mathrm{dpm} / \mathrm{ml}$ \\
\hline 5 & 680 & 138 & 4,440 & 612 & 312 & 32,940 & 10,290 \\
\hline 15 & 530 & 148 & 2,700 & 400 & 363 & 8,530 & 3,100 \\
\hline 29 & 320 & 149 & 1,880 & 280 & 330 & 5,160 & 1,700 \\
\hline 63 & 220 & 164 & 990 & 163 & 332 & 2,060 & 660 \\
\hline
\end{tabular}

1 The patient received glycine- $2-{ }^{14} \mathrm{C}$.

${ }^{2}$ FAM indicates the formaldemethone derivative of carbon 3 of serine. 

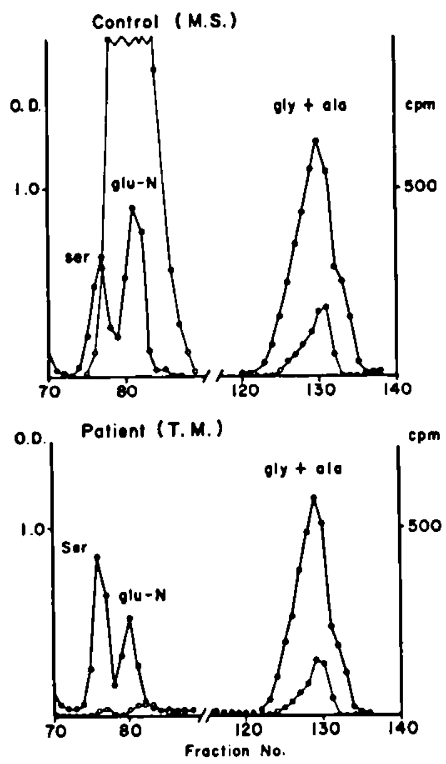

Fig. 2. Chromatographic analysis of the urine collected for $24 \mathrm{hr}$ following the administration of glycine-1 ${ }^{14} \mathrm{C}$. A dose of $2 \mu \mathrm{Ci} / \mathrm{kg}$ was administered intravenously to the control subject (MS) (2) and to the patient, TM. Aliquots of $3.0 \mathrm{ml}$ of urine were acidified and added to the $150-\mathrm{cm}$ column of the amino acid analyzer, which was operated in the usual fashion except that all of the cfluent was collected in 2-ml fractions. Aliquots of each fraction were analyzed by ninhydrin reaction and liquid scintillation counting (2). Ser: serine; glu-N: glutamine; gly + ala: glycine and alaninc; $\bullet$ : amino acid analysis by the ninhydric reaction. $O$ : radioactivity.

with ninhydrin. It was iclentified in column effuent using aliquots of $100 \mu \mathrm{l}$ subjected to paper chromatography on Whatman no. 1 paper using a solvent system of $n$-butanol-acetic acid-water $(4: 1: 1)$. Yellow spots were found at an $R_{F}$ of 0.85 after spraying with alka- line alcoholic bromcresol green. These spots were identical with those of authentic Na hippurate [30].

In control subjects receiving labeled glycine, isotope was regularly found in hippurate samples. In patient samples, conversion of glycine to hippurate was never observed.

\section{Detection of an Abnormal Conjugate of Glycine}

In order to obtain further information on the abnormal pattern of labeling of urinary hippurate the method clescribed by Chantrenne [6] was employed for the quantitation of hippurate. Acidified urine was extracted in $n$-butanol-chloroform. The solvent was evaporated. The residue was hydrolyzed in $2 \mathrm{~N} \mathrm{HCl}$ in a tightly sealed test tube. When the sealed test tube was opened, a peculiar, unpleasant odor emerged which was similar to that of a short-chain fatty acid. The hydrolysate was analyzed for glycine content on the amino acid analyzer. The chromatogram revealed an extremely large peak of glycine. An aliquot of the hydrolysate was also found to have a high level of radioactivity.

\section{Chromatography of the Abnormal Conjugate of Gly- cine}

The extract of urine prepared by the procedure for the determination of hippurate was applied to the 50. $\mathrm{cm}$ column of the automatic amino acid analyzer connected to a continuous flow analyzer system. A large radioactive peak appeared in the area just before taurine. The original urine was analyzed in the same manner. The results are illustrated in Figure 3 . In the illustration the large peak of radioactivity has been

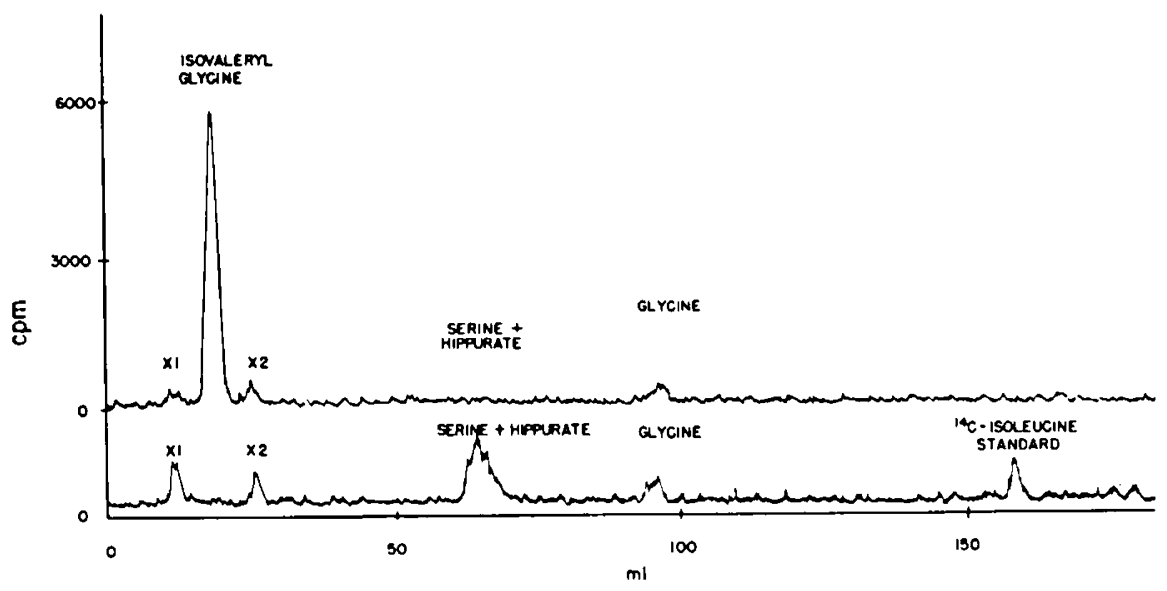

Fig. 3. Radiochromatograms of urinc from patient TM (above) and from a control subject (below). Each had been injected with glycine$1{ }^{14} \mathrm{C}$. Chromatography was carried out on the $50-\mathrm{cm}$ column of the automatic amino acid analyzer, and radioactivity was monitored con tinously in a flow meter. 

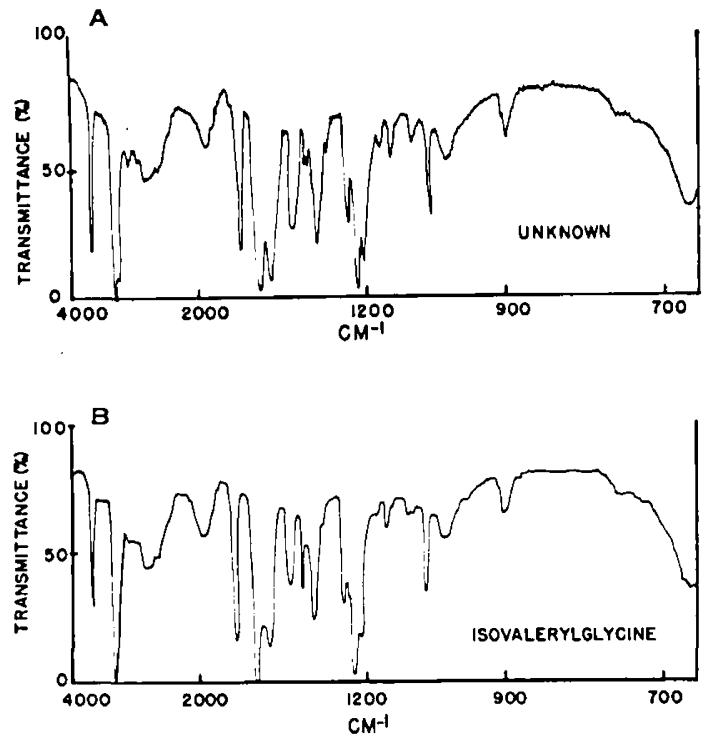

Fig. + Infrared spectra of the purified crystalline radioactive compound isolated from the urine of the patient $(A)$ and authentic isovalerylglycine $(B)$.

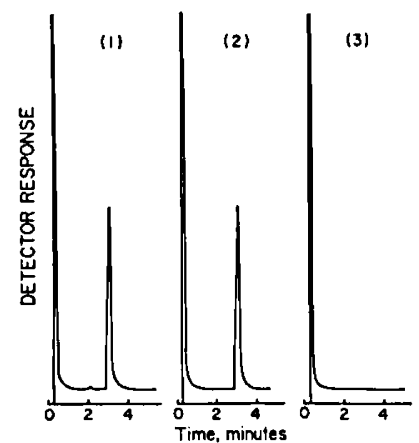

Fig. 5. Gas-liquid chromatograms of isovalerylglycine of the patient (1), the authentic compound (2), and the extract of the mother's urine (3). The $183 \cdot \mathrm{cm}$ by $4-\mathrm{mm}$ column was packed with $5 \%$ SE30. The oven temperature was $146^{\circ}$, and the flow rate of helium was $60 \mathrm{ml} / \mathrm{min}$. Attenuation was $10^{2} \times 64$. The extract equivalent to $5 \mu \mathrm{l}$ of urine was injected.

labeled, indicating its identification as isovalerylglycine. It was considerably larger than any other radioactive peak in either patient or controls. None of the urines from control individuals or from patients with nonketotic hyperglycinemia receiving ${ }^{14} \mathrm{C}$-glycine showed any radioactive peak in the area between the two small peaks $x_{1}$ and $x_{2}$ and before taurine. The labeled hippurate in the control and its absence in the patient samples are also illustrated. Similar amounts of labeled free glycine were found in patient and control samples. The amount of radioactivity excreted in the unknown compound was estimated to be $2.04 \mu \mathrm{Ci} / 24$ hr after injection of $34 \mu \mathrm{Ci}$ of ${ }^{14} \mathrm{C}$-glycine. Thus, as much as $6 \%$ of the isotope of glycine was incorporated into the unknown compound in the urine in $24 \mathrm{hr}$.

\section{Purification of the Abnormal Conjugate of Glycine}

An aliquot of urine was chromatographed as above, but the peak fractions were collected, pooled, and dried in vacuo. The dried sample was subjected to silicic acid column chromatography [19]. A radioactive peak was detected in aliquots and the remainder of each of the peak fractions was pooled and dried in a current of nitrogen. Crude crystals were obtained which were redissolved in ether and recrystallized by the addition of $n$-hexane.

\section{Identification of the Purified Crystalline Compound}

The purified crystals were ground into a dispersion with nujol for infrared analysis [31]. The spectrum of the unknown compound coincided completely with that of authentic isovalerylglycine (Fig. 4). It was therefore concluded that the unknown acylglycine was isovalerylglycine. In further confirmation of the identity of the unknown compound, it was subjected to gas-liquid chromatography (Fig. 5) [32]. The peak was identical to that of authentic isovalerylglycine. In addition, the mass spectrum of the crystalline compound was determined. The compound was applied directly to the solid sample inlet. The spectrum showed all of the characteristics of authentic isovalerylglycine.

\section{Quantitation of Isovalerylglycine in Urine}

The content of isovalerylglycine was then determined in urine. The acidified urine was extracted six times with ethylacetate. The extract was dried, and the residue was dissolved in ether-methanol and methylated [16]. Gas-liquid chromatography was performed [19] and the results are shown in Table II. The pa-

Table II. Quantitative assay of isovaleric acid and isovalerylglycine

\begin{tabular}{|c|c|c|c|}
\hline & \multicolumn{2}{|c|}{ Isovaleric acid } & \multirow{2}{*}{ 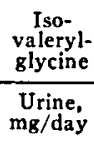 } \\
\hline & $\begin{array}{l}\text { Plasma, } \\
\text { mg/liter }\end{array}$ & $\begin{array}{l}\text { Urine, } \\
\text { mg/day }\end{array}$ & \\
\hline Patient & 2.86 & 2.56 & 2880 \\
\hline \multirow[t]{2}{*}{ Controls } & 0.58 & $0.40^{2}$ & $<2^{3}$ \\
\hline & $( \pm 0.22)^{1}$ & $0.36^{2}$ & \\
\hline
\end{tabular}

1 Values reported by Tanaka et al. (18).

2 Values obtained on two normal children 5 and 8 years of age.

3 Values reported by Tanaka et al. (19). 
tient excreted approximately $2.0 \mathrm{~g}$ of the compound in a 24-hr period.

\section{Quantitation of Isovaleric Acid in Plasma and Urine}

The detection of large amounts of isovalerylglycine in the urine of the patient indicated that she had isovaleric acidemia. It was of interest to document that there were elevated quantities of free isovaleric acid in body fluids.

The short-chain fatty acids in the plasma were determined by gas chromatography [18]. Isovaleric acid was present in a sizable amount in the plasma (Fig. 6). The concentration was about four to five times higher than that found in control individuals. Isovaleric acid was also quantitated in the urine using a simple gas chromatographic procedure. Urine was distilled by the method of Grant [9]. The distillate was titrated to $\mathrm{pH}$ 9 with $0.1 \mathrm{~N} \mathrm{NaOH}$ and dried. Sodium salts of volatile acids were redissolved in $50 \mu \mathrm{l} 0.3 \mathrm{~N} \mathrm{HCl}$ and injected directly into a $183-\mathrm{cm}$ by $4-\mathrm{mm}$ Porapak-QS column [29]. The resultant chromatogram is illustrated in Figure 7. Isovaleric acid was clearly demonstrable in the urine. Table II summarizes the quantities of isovaleric acid in the plasma and urine and of isovalerylglycine in the urine on a single day during remission. The values obtained in the patient were considerably ligher than those of controls and established an isovaleric acidemia. The differences between patient and controls in terms of isovaleric acid, however, were considerably smaller than the 1000 -fold difference in isovalerylglycine excretion.

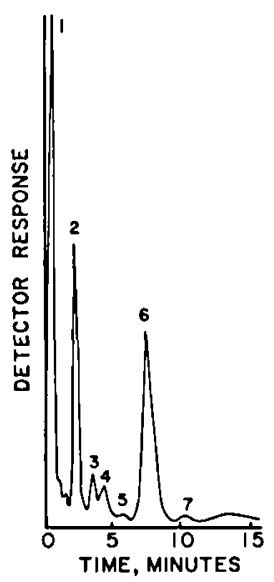

Fig. 6. Gas-liquid chromatogram indicating elevated isovaleric acid in the plasma of the patient. The method of Tanaka $e t$ al. [18] was employed. The numbers of the peaks represent peaks of solvent (1), acetic acid (2), propionic acid (3), isobutyric acid (4), butyric acid (5), isovaleric acid (6), and valeric acid (7).

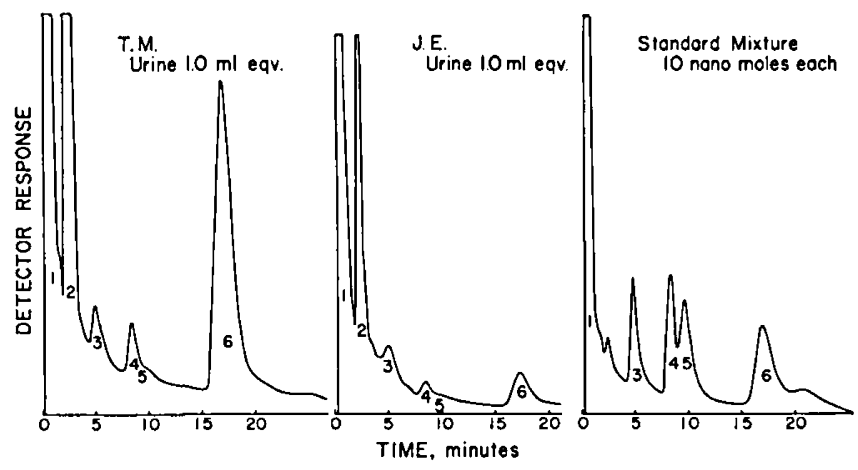

Fig. 7. Gas chromatograms of urinary short-chain fatty acids. The column used was a Porapak QS which was $183 \mathrm{~cm}$ by $4 \mathrm{~mm}$. The temperature of the column was $200^{\circ}$. The flow rate of the carrier gas was $50 \mathrm{ml} / \mathrm{min}$. The numbers represent the peaks of solvent (I), acetic acid (2), propionic acid (3), isobutyric acid (4), butyric acid (5), and isovaleric acid $(6) . T M$ is the patient. $J E$ is a patient with the Cornelia de Lange syndrome.

\section{Discussion}

Isovaleric acidemia is an inborn error of leucine metabolism in which elevated quantities of isovaleric acid are found in the blood [18]. Patients with isovaleric acidemia also excrete large amounts of isovaleric acid, isovalerylglycine, and $\beta$-hydroxyisovaleric acid in the urine [18-20]. The oxiclation of isotopically labeled isovaleric acid to $\mathrm{CO}_{2}$ by leukocytes is impaired in this condition [18]. A primary defect has been suggested in an enzyme, isovaleryl-CoA dehydrogenase [28], which catalyzes the conversion of isovaleryl-CoA to $\beta$-methylcrotonyl-CoA.

Very few patients with this disorder have as yet been described. Striking clinical features [5] include retardation of psychomotor development and an odor described as resembling that of sweaty feet, the odor of isovaleric acid. These patients suffer recurrent attacks of acidosis, vomiting, and dehydration leading to coma. An aversion to protein intake may have helped to protect some patients against the development of these attacks. Among the patients reported it may be possible to classify two subgroups on the basis of (1) time of onset of the first bout of acidosis or (2) the severity of symptoms. Some patients have had a relatively mild disorder characterized by intermittent acidosis, the unusual body odor, and mild retardation. The timing of the onset varied from early in the neonatal period to late in the lst year. The frequency of attacks of acidosis has varied considerably. The patient reported by Ulstrom [21] had a period of remission for as long as 2 years, lacked mental retardation, and nevertheless did not avoid protein. On the other hand, the 
young infant reported by Newman $c$ t al. [12] had overwhelming symptoms which led to early death. It is likely that other patients of this type have died without diagnosis. It is possible that there is a spectrum of presentation of a single disease. Apparent subgroups could reflect simply the factors which precipitate a catabolic state. It is also quite possible that the types of patients observed represent genetic heterogeneity.

Difficulty of diagnosis is indicated by the fact that even now it is not possible to detect an unusual body odor in our patient, and a negative history of the odor was attested to by the family and physicians. Certainly the history of the patient's brother suggests that his first bout of acidosis was fatal. He had been completely healthy before this attack at 13 months of age. The patient herself, on the other hand, had a relatively mild illness. Thus we have some evidence for two types of illness in the same family.

An odor reminiscent of sweaty feet has also been described by Sidbury, Smith, and Harlan [17] in a group of patients dying early in life with convulsions, lethargy, dehydration, and moderatc hepatomegaly. Leukopenia and thrombocytopenia were observed. Overwhelming illness very early in life and bouts of ketoacidosis, dehydration, and coma are also seen in what we have called ketotic hyperglycinemia or propionic acidemia and in methylmalonic acidemia. Neutropenia is also prominent in these two conditions. It has not previously been observed in isovaleric acidemia. The mechanism of the development of leukopenia in these conditions is not known. It does appear likely that it is common to a variety of these conditions.

Intolerance to certain branched-chain amino acids is a characteristic of propionic acidemia and methylmalonic acidemia as well as of isovaleric acidemia. Hyperglycinemia is a common feature of a number of these conditions, and its mechanism is unclear. Normal concentrations of glycine have been reported in isovaleric acidemia [5]. Our patient provides the first evidence that elevated quantities of glycine in body fluids may be seen in isovaleric acidemia.

The concentrations of glycine in the plasma of this patient had been as high as $3.2 \mathrm{mg} / 100 \mathrm{ml}$ at a time of good health and $4.2 \mathrm{mg} / 100 \mathrm{ml}$ during ketosis. This was interpreted as transient hyperglycinemia. The values at the time of study were lower. Glycine concentrations of $3-4 \mathrm{mg} / 100 \mathrm{ml}$ are not very high, but in this laboratory they are well above normal [7]. The glycinuria was much more impressive, and the $361 \mathrm{mg} / \mathrm{liter}$ noted was free glycine. Elevated amounts of glycine in both blood and urine indicate the presence of defective metabolism rather than a renal tubular defect. In addition, of course, significant quantities of glycine were being excreted as isovalerylglycine. These observations provide evidence of altered metabolism of glycine in this patient.

Isovaleric acidemia should probably be suspected in infants with unexplained acidosis and coma. Once suspected, the disease is not always easy to diagnose. During periods of remission the enormous elevations of isovaleric acid that lead to the unusual odor of the patient are not present. Isovaleric acid can be detected in the blood under these conditions with difficulty. The low concentrations of isovaleric acid observed during remission deserve emphasis. Patients with isovaleric acidemia usually have concentrations of isovaleric acid in the blood during remission which are only two to five times higher than normal [18]. On the other hand, the excretion of isovalerylglycine is extremely large in amount and relatively independent of the clinical condition of the patient or the blood level of isovaleric acid. The formation and excretion of isovalerylglycine can be considered a compensatory mechanism for the elimination of toxic amounts of isovaIeric acid from the body and the promotion of clinical remission. Tanaka and Isselbacher [19] recognized that isovalerylglycine is excreted in large amounts in isovaleric acidemia and that the presence of this compound in urine is unrelated to the remission or exacerbation status of the patient. Our experience suggests that the detection of isovalerylglycine may be the most convenient approach to the cliagnosis of isovaleric acidemia. In view of this, a simple screening method has been developed for the detection of isovalerylglycine in urine [1].

This patient may have demonstrated an intolerance to salicylate. The acidosis could well have been precipitated by the infection for which the salicylate had been given. Detoxification of acetylsalicylic acid (acetoxybenzoic acid), benzoic acid, and the short-chain fatty acids proceed via a common route [11, 15, 33]. The acylation step may be a common one for formation of isovalerylglycine from isovaleryl-CoA and glycine. These reactions proceed as follows:

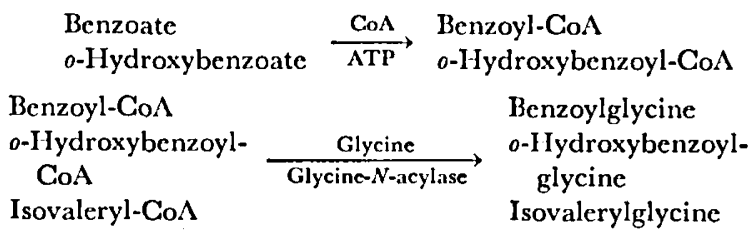

The formation of hippurate is usually limited by 
the amount of benzoic acid available. The labeling of hippuric acid after the injection of ${ }^{14} \mathrm{C}$-glycine was markedly diminished in the patient. The specific activity of the glycine pool as reflected by the glycine in the plasma which was measured and found to be normal indicated a normal pool size. Normal conversions to $\mathrm{CO}_{2}$ and serine were consistent with this. Diminished labeling of hippurate could be the result of a large pool of isovaleryl-CoA conjugating with glycine competitively. Administered glycine was largely utilized for formation of isovalerylglycine and to a very small extent for the formation of hippuric acid. Thus large amounts of isovaleryl-CoA appear to compete effectively for available amounts of glycine. It is an apparent discrepancy that glycine available for acyl glycine formation could be depleted without alteration in the general pool size or metabolism as measured by conversion to $\mathrm{CO}_{2}$ or serine. However, this is further evidence that there are at least two pools of glycine in the body, only one of which supplies the acyl glycine. Evidence was reported in 1953 by Weissbach and Sprinson [22] that the pool of glycine reflected in hippurate is a different one from that reflected in the free glycine of body fluids. Similarly, one might postulate that large amounts of salicylate might interfere with the formation of isovalerylglycine and lead to an enlarged pool of isovaleryl-CoA and isovaleric acid. In this way, salicylate administration could be harmful in this clisorder.

It is clear that what we have called ketotic hyperglycinemia is a clinical and chemical symptom complex which can be produced by a number of conditions including methylmalonic acidemia, propionic acidemia, and now isovaleric acidemia. We would predict that there will be others on this list. It should be recognized that hyperglycinemia is not always present in isovaleric acidemia. It can be useful in the diagnosis when present but is not a necessary requirement for the diagnosis of isovaleric acidemia.

\section{Summary}

The metabolism of glycine was studied in a patient with recurrent ketoacidosis associated with a modest elevation of plasma glycine, distinct hyperglycinuria, and leukopenia. Diminished labeling of urinary hippurate and an abnormal labeled compound were found after the intravenous injection of ${ }^{14} \mathrm{C}$-glycine. This compound was identified as isovalerylglycine. The diagnosis of isovaleric acidemia was confirmed by measurement of isovaleric acid in blood and urine.
The patient had no history of an unusual body odor. These observations indicate that isovaleric acidemia may induce alterations in glycine metabolism. Screening for isovalerylglycine may be the method of choice for the detection of isovaleric acidemia.

\section{References and Notes}

1. Ando, T., ANd Nyhan, W. L.: A simple screening method for detecting isovalerylglycine in urine of patients with isovaleric acidemia. Clin. Chem., 16: 420 (1970).

2. Ando, T., Nyhan, W. L., Gerritsen, T., Gong, L., Heiner, D. C., AND BRAY, P. F.: Metabolism of glycine in the nonketotic form of hyperglycinemia. Pediat. Res., 2: 254 (1968).

3. Ando, T., Rasmussen, K., Nyihan, W. L., Donnel., G., ANd BARNes, N. D.: Propionic acidemia in patients with ketotic hyperglycinemia in remission. J. Pediat., 78: 827 (1971).

4. BONDI, S., AND EISSLER, F.: Uber Lipoproteide und die Deutung der degenerativen Zellverfettung. V. Weitere Synthesen von Lipopeptiden. Biochem. Z., 23: 499 (1910).

5. Budd, M. A., Tanaka, K., Holmes, L. B., Efron, M. L., CrawFORD, J. D., AND IsselbaCIIER, K. J.: Isovaleric acidemia. Clinical features of a new genetic defect of leucine metabolism. New Engl. J. Med., 277: 321 (1967).

6. Chantrenne, H.: Hippuric acid synthesis. In: S. P. Colowick and N. O. Kaplan: Methods in Enzymology, Vol. II, p. 346 (Academic Press, New York, 1955).

7. Childs, B., Nyhan, W. L., Borden, M., Bard, L., and Cooke, R. E.: Idiopathic hyperglycinemia and hyperglycinuria, a new disorder of amino acid metabolism. Pediatrics, 27: 522 (1961).

8. Gompertz, D., Storrs, C. N., Bay, D. C. K., Peters, T. J., ANd Hugnes, E. A.: Localisation of cnzymic defect in propionic acidacmia. Lancet, $i: 1140$ (1970).

9. Grant, W. M.: Apparatus for quantitative low-temperature vacuum distillation of milliliter volumes. Ind. Eng. Chem. Anal. Ed., 18: 729 (1946).

10. Honmes, F. A., Kuirers, J. R. G., Elema, J. D., Jansen, J. F., AND Jonxis, J. H. P.: Propionic acidemia, a new inborn error of metabolism. Pediat. Res., 2: 519 (1968).

11. MAhleR, II. R., WAKIL, S.J., AND Bock, R. M.: Studics on fatty acid oxidation. I. Enzymatic activation of fatty acids. J. Biol. Chem., 20t: 453 (1953).

12. Newman, C. G. H., Wilson B. D. R., Calllagiham, P., aNd Yound, L.: Neonatal death associated with isovaleric acidemia. Lancet, ii: 439 (1967).

13. Oberholzer, V. G., Levin, B., AnN Burgess, E., and Young, W. F.: Methylmalonic aciduria. An inborn cror of metabolism leading to chronic metabolic acidosis. Arch. Dis. Childhood, 42: 492 (1967).

14. Rasmussen, K., Ando, T., and Nyhan, W. L.: A simple gas chromatographic detection of short chain fatty acids in biological fluids. To be published.

15. Schachter, D., and Taggart, J. V.: Glycine $N$-acylase: Purification and properties. J. Biol. Chem., 208: 263 (1954).

16. Schlenk, H., and Gellerman, J. L.: Esterification of fatty acid with diazomethane on a small scale. Analyt. Chem., 32: 1412 (1960).

17. Sidbury, J. B., JR., Smith, E. K., ANd Harlan, W.: An inborn error of short-chain fatty acid metabolism. J. Pediat., 70: 8 (1967).

18. Tanaka, K., Budd, M. A., Efron, M. L., ANd Isselbacher, 
K. J.: Isovaleric acidemia: A new genetic defect of leucine metabolism. Proc. Nat. Acad. Sci. U. S. A., 56: 236 (1966).

19. TANAKA, K., AND Isselbacifer, $K$. J.: The isolation and identification of $N$-isovalerylglycine from urine of patients with iso. valeric acidemia. J. Biol. Chem., 242: 2966 (1967).

20. Tanaka, K., OrR, J. C., and Isselbacher, K. J.: Identification of $\beta$-hydroxyisovaleric acid in the urine of a patient with isovaleric acidemia. Biochim. Biophys. Acta, 152: 638 (1968).

21. Ulstrom, R. A.: In: A discussion of isovaleric acidemia. Abstracts of the Socicty for Pediatric Research, Atlantic City, 1966. J. Pediat., 69: 1961 (1966).

22. Weissbach, A., and Sprinson, D. B.: Metabolism of 2-carbon compounds related to glycine. J. Biol. Chem., 203: 1023 (1953).

23. Williams, R. T.: Detoxification Mechanism, p. 359 (Wiley, New York, 1959).

24. Spinco Division, Beckman Instruments, Palo Alto, Calif.

25. New England Nuclear, Boston, Mass.

26. Nuclear-Chicago, Chicago, Ill.

27. Tri-Carb, Packard Instrument Co., Downers Grove, Ill.

28. Model 1402, Hewlett-Packard Co., Palo Alto, Calif.
29. Porapak-QS, Water Asssoc., Framingham, Mass.

30. Mann Research Laboratories, New York, N. X.

31. Model 137, Perkin-Elmer Corp., Norwalk, Conn.

32. Model 1015, Finnigan Instruments, Palo Alto, Calif.

33. These experiments were undertaken in accordance with institutional regulations on human experimentation. Written evidence of informed consent has been obtained.

34. We acknowledge the assistance of Drs. John E. Jones and E. B. Flink, Directors of the Clinical Center for Metabolic Studies of the West Virginia University Hospital.

35. Supported by Public Health Service Research Grant no. HD04608 from the National Institute of Child Health and Human Development and by Grant no. AM 05578-07 AMP from the National Institute of Arthritis and Metabolic Diseases, National Institutes of Health.

36. Request for reprints should be addressed to: Willian $L$, NyunN, M.D., Ph.D., Department of Pediatrics, University of California, San Diego, P. O. Box 109, La Jolla, Calif. 92037 (USA).

37. Accepted for publication November 18, 1970. 